\title{
Medulloblastoma - clinical and therapeutical aspects
}

\author{
Catalin Prazaru', Georgeta Diaconu', \\ Ana Ulinici ${ }^{1}$, Husamedin Abou Ainain ${ }^{1}$, Alexandra Stoicescu Prazaru², Ioana Grigore ${ }^{1}$ \\ ${ }^{1}$ Pediatric Neurology Department, Children's Emergency Hospital "St. Mary", Iasi, Romania \\ ${ }^{2}$ Radiation Oncology Department, Regional Oncology Institute, Iasi, Romania
}

\begin{abstract}
Medulloblastoma is a primitive neuroectodermal tumor commonly seen in pediatric age, with peak incidence between 5 and 10 years. The tumor is generally located in the vermis or cerebellar hemispheres invading IV ventricle. Symptomatology is dominated by signs of intracranial hypertension and cerebellar ataxia. Neuroimaging explorations contributes to diagnosis and more precise staging medulloblastoma. The treatment is complex including surgical resection of the tumor followed by radiotherapy and chemotherapy. The prognosis is generally reserved, incidence of neurological sequelae after treatment is high.
\end{abstract}

Keywords: medulloblastoma, child

Medulloblastoma (MBL) is a primary embryonic tumor of cerebellum, considered the most common intracranial child malignant tumor representing approximately $25 \%$ of all malignancies of the central nervous system (CNS) before the age of 15 years (1). Was described for the first time in 1925 by Cusing and Bailey as a cerebellar small cell tumor and high malignancy (2). Later, in 1983, Rorke, Becker and Hinton they have proposed that medulloblastoma be included, along with all intracranial tumors of small cell in the category of primitive neuroectodermal tumors (3). Currently, no longer considered similar entities as opposed to MBL, supratentorial primitive neuroectodermal tumors have a distinct genetic profile, they are usually more aggressive and respond poorly to specific therapy. For these reasons, in the fourth edition of embryonic CNS tumors classification, published by the World Health Organization in 2007, MBL remains a distinct category of other primitive neuroectodermal tumors (3).

Is usually located in vermis and fourth ventricle with often cerebellar hemispheres invading. The tumor has a vicinity extension character and a ten- dency to disseminate in subarachnoid spaces, particularly at spinal region (1).

Medulloblastoma is rare in adults, $75 \%$ of cases are diagnosed in children under 16 years old (4). The literature reports a male predominance of the disease 2:1 (5).

\section{HISTOPATHOLOGY}

Medulloblastoma originates from immature or embryonal cells at their earliest stage of development and the cell of origin depends on the subgroup of medulloblastoma. Both perivascular pseudorosette and Homer Wright rosette pseudorosettes formation are highly characteristic of medulloblastoma and is seen in up to half of the cases. Homer Wright rosettes are pseudorosettes consisting of tumor cells surrounding a fibrillar area (6). In 2004 Eberhart classified into 3 types histopathological MBL: classic or undifferentiated, anaplastic, or nodular desmoplastic (6). This tumor may have several aspects in terms of histopathological design. Genomic and molecular recent studies have established that MBL presents four distinct molec- 
ular variants (7). Studies have shown that each molecular subgroup have particular demographic and clinical characteristics $(7,8)$.

Medulloblastoma type wingless is the most rare form, being diagnosed in about $10 \%$ of children (9). It can occur at any age but is less than 3 years old. Gender distribution is equal. The tumor is generally located on the midline and occupies fourth ventricle. The rate of metastasis and recurrence risk is low, 5-year survival of these patients is $95 \%(10)$.

Medulloblastoma type sonic hedgehog represents approximately $30 \%$ of total MBL, being more frequent in the age group 0-3 years and children $>$ 16 years. Risk of metastasis is low, but local recurrences may occur. Prognosis depends on the age of onset, beeing better at younger age with a 5-year survival of $75 \%$ (9).

Medulloblastoma grup 3 is diagnosed in approximately $25 \%$ of the cases, is common in males, generally occurs in infants and children, rarely in adolescents and never in adults (11). The risk of metastasis is higher, approximately $50 \%$ of patients have metastases (12). The prognosis is poor with a 5 -year survival rate between 45 and 58\% $(11,13)$.

Medulloblastoma grup 4 is the most common form of MBL, beeing diagnosed in almost $35 \%$ of MBL cases (9). It occurs at any age, but less common in infants. This subgroup is more frequent in males with gender distribution 3:1 (11). The risk of metastasis is higher and almost $35-40 \%$ of patients have metastases $(11,13)$. 5-year survival rate is between 60 and $80 \%$ (14).

Kool (2012) considers that the molecular MBL classification system can improve the way of treatment, having an important role in case prognosis (13).

Clinical symptoms are varied and depends on age, neuromotor development of the child, the lo- cation and extent of the tumor. Early signs not specific as irritability, decreased academic skills and fatigue are generally ignored by the patient or their family. In 2012 the study of Brasme and collaborators (5) have demonstrated the existence of an average delay between the first signs of MBL and diagnostic of approximately 65 days late which was significantly higher in children presenting with psychiatric symptoms (low school, behavioral disorders and mood swings).

Because MBL develops generally close to IV ventricle and it may extend to ventricular cavity blocking the normal route of cerebrospinal fluid causing hydrocephalus and signs of intracranial hypertension. In a study by Muzumdar (2007) of 154 patients diagnosed with MBL, $96.5 \%$ of them have hydrocephalus (15). Also Kombogiorgas (2008) reported the presence of hydrocephalus in 20 patients with MLB followed by him (16). In older children clinical signs are due to increase intracranial pressure and it is represented by headache, vomiting, visual disturbances; in infants by macrocranie, bulging fontanelle, irritability. Torticollis can be the first symptom of $10 \%$ patients (15).

In the course of illness can appear impaired general condition with disorder movements of the eyes, amblyopia and cerebellar ataxia. In almost 10-35 \% of patients spinal metastases signs are present from the time of diagnoses and it generally manifests by motor deficits, radicular pain (17).

\section{DIAGNOSIS}

The first step in the diagnosis is a complete neurological examination and funduscopic evaluation.

Funduscopic evaluation may reveal papilledema in $90 \%$ of cases $(18)$.
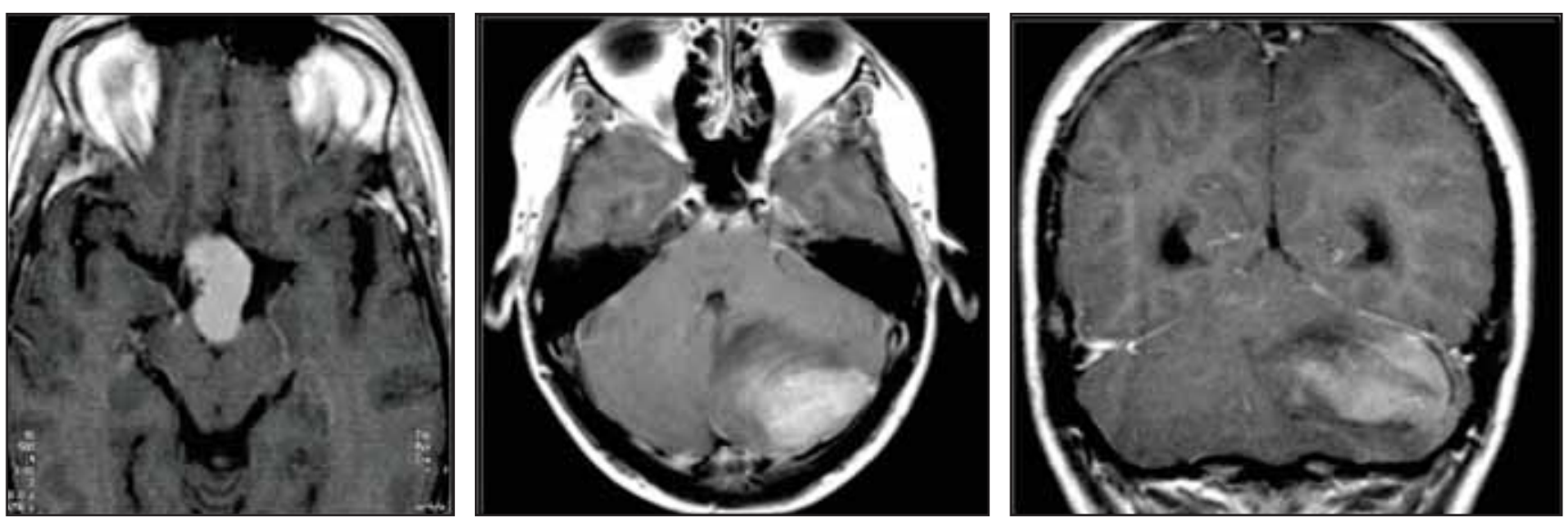

FIGURE 1. Medulloblastoma. MRI axial and coronal section:inhomogeneous mass with white matter extension to left cerebellar hemisphere. (Pediatric Neurology Department Collection) 
The most important way of diagnosis is the high performance neuroimaging evaluation. CT - scan and brain MRI, may show a round lesion on the middline that captures contrast, often located in vermis with invasion on fourth ventricle and an active hydrocephalus (Fig. 1). Given the tendency of this tumor to disseminate throughout the central nervous system early in the course of illness, MRI of the entire brain and spinal cord is indicated. Neuroimaging evaluation also helps on tumor staging with an important role of therapeutic decisions (Table 2).

TABLE 2. Neuroimaging staging of medulloblastoma

\begin{tabular}{|l|l|}
\hline T1 & Tumor $<3 \mathrm{~cm}$ in diameter \\
\hline T2 & Tumor $>3 \mathrm{~cm}$ in diameter \\
\hline T3A & $\begin{array}{l}\text { Tumor }>3 \mathrm{~cm} \text { in diameter with spread to nearby } \\
\text { structures }\end{array}$ \\
\hline T3B & $\begin{array}{l}\text { Tumor }>3 \mathrm{~cm} \text { in diameter with def nite spread into the } \\
\text { brain stem }\end{array}$ \\
\hline T4 & $\begin{array}{l}\text { Tumor }>3 \mathrm{~cm} \text { in diameter with extension up past the } \\
\text { aqueduct of Sylvius and/or down past the foramen } \\
\text { magnum }\end{array}$ \\
\hline M0 & No evidence of metastasis \\
\hline M1 & $\begin{array}{l}\text { Tumor cells found in cerebrospinal f uid (by lumbar } \\
\text { puncture and cytology study) }\end{array}$ \\
\hline M2 & Tumor beyond primary site but still in brain \\
\hline M3 & $\begin{array}{l}\text { Tumor deposits ("seeds") in spine area that are easily } \\
\text { seen on MRI }\end{array}$ \\
\hline M4 & $\begin{array}{l}\text { Tumor spread to areas outside the CNS (outside both } \\
\text { brain and spine) }\end{array}$ \\
\hline
\end{tabular}

Because MBL are very rarely metastatic to the bone or other body sites at the time of diagnosis, studies such as chest $\mathrm{x}$-rays, abdominal ultrasound or bone scans are not indicated, unless there are symptoms or signs suggesting organ involvement.

Cytological examination of cerebrospinal fluid is useful to diagnose microscopic meningeal dissemination.

The final diagnoses is based on the pathological examination.

Treatment of this disease is complex and includes four steps: surgical resection, radiotherapy, chemotherapy, supportive and recovery therapy.

Surgery is typically the first component of therapy. It is very important to perform as complete a surgery as possible, with the goal being removal of all visible tumors, while sparing as much surrounding brain tissue as possible. Collange et al. (2016) consider that almost $32.45 \%$ of patients with MBL require shunt to drain cerebrospinal fluid after surgical removal of the tumor (19). Kombogiorgas (2008) reported that $20 \%$ of patients with surgical resection needed a sistemic shunt to drainage cerebrospinal fluid (16). Also, Gopalakrishnan (2012) showed that $36.8 \%$ of 38 patients with MBL surgery need to drainage cerebrospinal fluid by sistemic shunt (20).

Wang (2013) consider that the prognosis depends on the resection of the tumor (12). Monteight (2006) studied 36 patients with MBL, 16 of them with complete tumor resection, 14 with partial resection, and 6 with biopsy; he observed that those 16 patients with complete surgical resection of the tumor had a greater survival rate (21). A post-operative MRI scan of the brain to look for any leftover or residual tumor always be carried out in the first 24-72 hours after surgery. Nowadays, reported mortality after surgical resection of MBL approaches to 0 , but neurological sequelae incidence increased up to $57 \%$, most common being represented by cerebellar ataxia and palsy of oculomotor nerves which may improve later on (22).

Radiation therapy is an effective adjuvant treatment in MBL and it applies to brain and spinal cord too, even if there are not identified any distance disemination. The main purpose of radiotherapy is to prevent local recurrences (19). Used doses are 3540 Gy for spinal axis and 55-60 Gy for tumor bed, macroscopic residual tumor and metastasis (23). Rutkowski (2009) believes that radiotherapy should be avoided at less than 3 years old children (24). This idea is supported by the findings of LafayCousin (2009) who evaluated 29 children of 3 years old or younger diagnosed with MBL, and noted that those who received radiation therapy has been developed a significant drop in IQ and school performance than those in which this method of treatment has been delayed (25). Also, Thakur et al. (2016) observed that $20 \%$ of 52 pediatric patients diagnosed with MBL who only benefited from radiotherapy as adjuvant treatment, had acute haematological toxicity that was detected during radiotherapy, but also after its interruption (26).

Chemotherapy may be used to treat this type of tumor because is usually chemosensitive. On children less than 3 years old,chemotherapy is the main adjuvant therapy because improves patient survival and delay the start of radiotherapy, which has multiple long-term important side effects. In literature there are different protocols, the most commonly used are lomustine, cisplatin, carboplatin, vincristine and cyclophosphamide in various combinations (26).

Supportive and neurological rehabilitation therapy has an important role in long-term management of treatment. These actions aim to improve mental and neuromotor deficits, which are unfortunately very common on these patients. 


\section{PROGNOSIS}

In the ninetheen's, 10-year survival rate of children with meduloblastoma was $50 \%$. Currently, by modern treatment techniques, $50-60 \%$ of pediatric operated patients have tumor progression at 5 years after diagnosis. Multi-agent chemotherapy combination in postoperative children with part of the tumor on site, to increased 5-year survival rate to $80 \%$ (27). Some authors consider that parameters such as age, sex, extent of lesion and histopathological variant do not significantly affect the survival rate (29).

The prognosis is influenced by the presence of neuropsychiatric and endocrine sequelae that may be present in such patients and which are due to direct and indirect tumor effects, surgery, chemotherapy and radiotherapy. Patel (2015) supervised for 36-58 months a group of 100 children aged 3-14 years diagnosed with MBL who received surgical treatment associated with radiotherapy and chemotherapy, reporting the presence of neurological deficits in $63 \%$ of patients and endocrine problems in $58 \%$ (30). Other studies reported in children with

\section{REFERENCES}

1. Ashley D., Lyon N., Bonner M. et al. Developmental and functional outcome following postoperative chemotherapy and local conformal radiation in infants with medulloblastoma using a centrally administered phone-based interview technique, initial report: a Children's Oncology Group study. Neuro-oncol. 2008; 45: 434.

2. Bailey P., Cushing H. Medulloblastoma cerebelli: a common type of medicerebellar glioma of childhood. Arch Neurol Psychiatry. 1925; 14:192-224.

3. Louis D.N., Ohgaki H., Wiestler O.D. et al. The 2007 WHO classification of tumours of the central nervous system. Acta Neuroathol. 2007; 114:97-109.

4. Smee R.I., Williams J.R., De-Loyde K.J. et al. Medulloblastoma: progress over time. J Med Imaging Radiat Oncol. 2012; 56(2):227-34.

5. Brasme J.F., Chalumeau M., Doz F. et al. Interval between onset of symptoms and diagnosis of medulloblastoma in children: distribution and determinants in a population-based study. Eur J Pediatr. 2012; 171: 25-32.

6. Eberhart C.G., Kratz J., Wang Y. et al. Histopathological and molecular prognostic markers in medulloblastoma: C-myc, N-myc, TrkC and anaplasia. J Neuropathol Exp Neurol. 2004; 63(5):4441-4449.

7. Northcott P.A., Korshunov A., Witt H. et al. Medulloblastoma comprises four distinct molecular variants. J Clin Oncol. 2011; 29:1408-1414.

8. Robinson G., Parker M., Kranenburg T.A. et al. Novel mutations target distinct subgrups of medulloblastoma. Nature. 2012; 488:43-48.

9. Kijima N., Kanemura Y. Molecular Classification of Medulloblastoma. Neurol Med Chir (Tokyo). 2016;56:687-697.

10. Taylor M.D., Northcott P.A., Korshunov A. et al. Molecular subgrups of medulloblastoma: the current consensus. Acta Neuropathol. 2012; 123:465-72.

11. Northcott P.A., Korshunov A., Pfister S.M., Taylor M.D. The clinical implications of medulloblastoma subgroups. Nat Rev Neurol. 2012; 8:340-351
MBL numerous posttherapeutic side effects such as lowering IQ, visual disturbances, changes in adaptive behavior, neuromotor deficits (27). Maddrey (2005) have evaluated 16 children with medulloblastoma by neuropsychological tests, at 10 years from the diagnosis and therapeutic intervention and he reported values between 54 and 110 IQ with average of 75 (28). Also, Philip et al. (2016) noticed a neurocognitive decline in $48 \%$ of children diagnosed with MBL who received surgical treatment followed by radiotherapy and chemotherapy (29).

\section{CONCLUSIONS}

Medulloblastoma is a malignant tumor common in infants and young children with symptoms resulting on intracranial hypertension and cerebellar ataxia. Neuroimaging evaluation is the main poin for diagnose and staging of this disease. Nowadays, the multimodal treatment has significantly improved the prognostic of those children. Supportive and neurorecovery therapy has an important role to improve quality of life of these patients.

\section{Conflict of interest: none declared Financial support: none declared}

12. Wang X., Ramaswamy V., Remke M. et al. Intertumoral and intratumoral heterogeneity as a barrier for effective treatment of medulloblastoma. Neurosurgery. 2013; 60 (Suppl 1):57-63.

13. Kool M., Korshunov A., Remke M. et al. Molecular subgroups of medulloblastoma: an international meta-analysis of transcriptome, genetic aberrations and clinical data of WNT, SHH, Group 3 and Group 4 medulloblastomas. Acta Neuropathol. 2012; 123:473-484.

14. Shih D.J., Northcott P.A., Remke M. et al. Cytogenetic prognostication within medulloblastoma subgroups. J Clin Oncol. 2014; 32:886-896.

15. Muzumdar D.P. Ventricular CSF drainage and medulloblastoma. Ped Neurosurg. 2007; 43(1):74-5.

16. Kombogiorgas D., Natarajan K., Sgouros S. Predictive value of preoperative ventricular volume on the need for permanent hydrocephalus treatment immediately after resection of posterior fossa medulloblastomas in children. J Neurosurg Pediatr. 2008; $1(6): 451-5$.

17. Agerlin N., Gjerris F., Brincker H., Haase J., Laursen H., Møller K.A., Ovesen N., Reske-Nielsen E., Schmidt K. Childhood medulloblastoma in Denmark 1960-1984. A population-based retrospective study. Childs Nerv Syst. 1999 Jan; 15(1):29-36.

18. Winn H.R. Youmans Neurological Surgery. 6th edition. Saunders, 2011

19. Collange N.Z., de Azevedo Brito S., Campos R.R. et al. Treatment of medulloblastoma in children and adolescents. Rev Assoc Med Bras. 2016; 62(4):298-302.

20. Gopalakrishnan C.V., Dhakoji A., Menon G., Nair S. Factors predicting the need for cerebrospinal fluid diversion following posterior fossa tumor surgery in children. Pediatr Neurosurg. 2012; 48(2):93-101.

21. Monteith S.J., Heppner P.A., Woodfield M.J. et al. Paediatric central nervous system tumours in a New Zealand population: a 10-year 
experience of epidemiology, management, strategies and outcomes. J Clin Neurosci. 2006; 13(7):722-9.

22. Sanders R.P., Onar A., Boyett J.M. et al. M1 medulloblastoma: high risk at any age. J Neurooncol. 2008; 90:351-355.

23. Gajjar A., Hernan R., Kocak M. et al. Clinical, histopathologic and molecular markers of prognosis: toward a new disease risk stratification system for medulloblastoma. J Clin Oncol, 2004, 22 (6), 984-993.

24. Rutkowski S., Gerber N.U., von Hoff K. et al. German Pediatric Brain Tumor Study Group. Treatment of early childhood medulloblastoma by postoperative chemotherapy and deferred radiotherapy. Neuro Oncol. 2009; 11(2):201-10.

25. Lafay-Cousin L., Bouffet E., Hawkins C. et al. Impact of radiation avoidance on survival and neurocognitive outcome in infant medulloblastoma. Curr Oncol. 2009; 16(6):21-8.

26. Thakur P., Kumar N., Miriyala R., Ghoshal S. Cranio-spinal irradiation - is acute hematological toxicity under-reported? A single institutional experince. Ann Oncol. 2016; 27(suppl 9):42-45.
27. Grill J., Sainte-Rose C., Jouvet A. et al. Treatment of medulloblastoma with postoperative chemotherapy alone: an SFOP prospective trial in young children. Lancet Oncol. 2005; 6:573-580.

28. Wells E.M., Walsh K.S., Khademian Z.P. The cerebellar mutism syndrome and its relation to cerebellar cognitive function and the cerebellar cognitive affective disorder. Dev Disabil Res Rev. 2008; 14:221-228.

29. Philip A., Munirathnam V., Pavithran K., Wesley J., Poorna D., Pillai R. Treatment outcome in medulloblastoma with the POG 9031 protocol: a single institution review. Ann Oncol. 2016; 27(suppl 6):334P.

30. Patel P.N., Vyas R., Mehta M. Quality of life of pediatric patients with medulloblastoma: Results from single regional cancer centre. J Clin Oncol. 2015; 33 (suppl):21038.

31. Maddrey A.M., Bergeron J.A., Lombardo E.R et al. Neuropsychological performance and quality of life of 10 year survivors of childhood medulloblastoma. J Neurooncol. 2005; 72:245-53. 